\title{
Fenchel Problem of Level Sets
}

\section{T. Rapcsák}

Published online: 2 April 2008

(C) Springer Science+Business Media, LLC 2008

\section{Erratum to: J Optim Theory Appl DOI 10.1007/s10957-005-6399-y}

In Ref. [1], the correct form of the solution of the Fenchel problem of level sets is as follows:

Theorem 3.1 Let $f \in H_{l L}$ be a real-valued function defined on an open convex set $A \subseteq R^{n}$. Then, $f$ is convex image transformable by a one-to-one increasing function $\phi \in C^{2}\left(\operatorname{Im}_{f}(A), R\right)$ iff, for every $\mathbf{x} \in A$, there exists a convex neighborhood $U(\mathbf{x}) \subseteq$ A such that, for every pair $(\mathbf{x}, \mathbf{y}=\mathbf{z}-\mathbf{x}), \mathbf{z} \in A$, the single variable function

$$
f\left(\mathbf{x}+\varphi_{(\mathbf{x}, \mathbf{y})}(t) \mathbf{y}\right), \quad \mathbf{x}+\varphi_{(\mathbf{x}, \mathbf{y})}(t) \mathbf{y} \in U(\mathbf{x}), t \in[0,1],
$$

is convex, where

$$
\varphi_{(\mathbf{x}, \mathbf{y})}:[0,1] \rightarrow R, \quad \varphi_{(\mathbf{x}, \mathbf{y})}(0)=0, \quad \varphi_{(\mathbf{x}, \mathbf{y})}^{\prime}(0)=1,
$$

is a strictly increasing function given by the following differential equation:

$$
\varphi_{(\mathbf{x}, \mathbf{y})}^{\prime}(t)=\frac{1}{\phi^{\prime}(f(\mathbf{x}))} \phi^{\prime}\left(f\left(\mathbf{x}+\varphi_{(\mathbf{x}, \mathbf{y})}(t) \mathbf{y}\right)\right), \quad t \in[0,1] .
$$

Moreover, if $\phi: A \rightarrow R_{+}$and

$$
\nabla f(\mathbf{x}) \mathbf{y}>0
$$

then $\varphi_{(\mathbf{x}, \mathbf{y})}$ is strictly convex.

The online version of the original article can be found under doi:10.1007/s10957-005-6399-y.

T. Rapcsák $(\bowtie)$

Computer and Automation Research Institute, Hungarian Academy of Sciences, Budapest, Hungary

e-mail: rapcsak@oplab.sztaki.hu 
In the proof, the formulas (11) and the ones after (13), (14), (25), (26), (39), (40), (41) contain misprints.

\section{References}

1. Rapcsák, T.: Fenchel problem of level sets. J. Optim. Theory Appl. 127, 177-191 (2005) 